\title{
Collision Tumor Composed of Meningioma and Cavernoma
}

\author{
Jens Weigel, M.D., ${ }^{1}$ Markus Neher, M.D., ${ }^{1}$ Michael Schrey, M.D., Peter H. Wünsch, M.D., Ph.D., ${ }^{2}$ Hans-Herbert Steiner, M.D., Ph.D. \\ Department of Neurosurgery, Institute of Pathology, Paracelsus Medical University, General Hospital Nuremberg, Nuremberg, Germany
}

A true collision tumor is a rare entity composed of two histologically distinct neoplasms coinciding in the same organ. This paper reports a unique case of cerebral collision tumor consisting of two benign components. On the first hand, meningioma which is usually a benign lesion arising from the meningothelial cell in the arachnoidal membrane. On the other, cerebral cavernoma which is a well-circumscribed, benign vascular hamartoma within the brain. To our knowledge, there is no previously documented case of cerebral collision tumor consisting of two benign components. A 56-year-old Caucasian male suffered in 2002 from an atypical meningioma $\mathrm{WHO} \|^{\circ}$ located in the left lateral ventricle. Three years after the tumor extirpation, the patient suffered from a hematoma in the fourth ventricle due to a recurrently haemorrhaged cavernoma. In 2008, a recurrence of the tumor in the left lateral ventricle was discovered. Additionally, another tumor located in the quadrigeminal lamina was detected. After surgical resection of the tumor in the left lateral ventricle, the pathological examination confirmed the diagnosis of a collision tumor consisting of components of a meningioma $\mathrm{WHO} \mathrm{II}^{\circ}$ and a cavernoma. Postoperatively, no adjuvant treatment was needed and no tumor recurrence is discovered up to the present. A possible explanation for the collision of those two different tumors may be migration of tumor cells mediated by the cerebrospinal fluid. After 5-years of follow-up, there is no sign of any tumor recurrence; therefore, surgical tumor removal without adjuvant therapy seems to be the treatment of choice.

Key Words : Collision tumor $\cdot$ Meningioma $\cdot$ Cavernoma $\cdot$ Tumor intraventricular.

\section{INTRODUCTION}

A true collision tumor represents a coexistence of two adjacent but histologically different malignant neoplasm's occurring in the same organ without histological admixture or an intermediate cell population zone ${ }^{11)}$. Such tumors consist of components with different histogenesis and different tumorigenic pathway representing a mosaic of two concurrent but independent tumors that have "collided" with each other. Thus, collision tumors are synchronous morphologi- cally different neighboring neoplasms that have expanded into each other's territory in the same organ. This paper, reports a unique case of cerebral collision tumor consisting of two benign components. To our knowledge, this is the first documented case of such a collision tumor. Additionally, this case report shows the importance of detailed clinical history in diagnosing a tumor composed of multiple morphologies as well as choosing the best treatment.

- Received: July 7, 2015 •Revised: December 8, 2015 •Accepted: December 10, 2015

- Address for reprints : Jens Weigel, M.D.

Department of Neurosurgery, Paracelsus Medical University, General Hospital Nuremberg, Breslauer Str. 20190471 Nuremberg, Germany Tel : +49-911-398 2318, Fax : +49-911-398 2981, E-mail : jens.weigel@klinikum-nuernberg.de

This is an Open Access article distributed under the terms of the Creative Commons Attribution Non-Commercial License (http://creativecommons.org/licenses/by-nc/4.0) which permits unrestricted non-commercial use, distribution, and reproduction in any medium, provided the original work is properly cited. 


\section{CASE REPORT}

A 56-year-old Caucasian male suffered from non-traumatic severe headache and intermitting nausea. An magnetic resonance imaging (MRI) of the brain revealed a tumor located in the posterior horn of the left lateral ventricle. In 2002, a first surgical extirpation of the tumor was performed at an external hospital. The histological examination including immunohistochemical profile showed an atypical meningioma WHO $\mathrm{II}^{\circ}$ (Fig. 1, 2).

During a prolonged postoperative reconvalescence, the patient suffered from a heparine induced thrombocytopenia II combined with pulmonary embolism, deep venous thrombosis and basal ganglion infarction with consecutive right sided hemiparesis. Two years after the meningioma extirpation, the patient suffered from recurring headaches, dizziness and ataxia. Cerebral CT-scan showed a hydrocephalus for which ventriculoperitoneal shunting was performed in 2004. After this procedure, the patient recovered well.

In 2005 , the patient was re-referred to the same external hospital because of recurrent headaches and dizziness. Imaging-studies showed a hematoma in the fourth ventricle due to a haemorrhaged tumor located in the caudal section of the aqueduct and dorsolaterally to the fourth ventricle. After tumor removal in 2005, the pathological examination revealed a recurrently haemorrhaged cavernoma with regressive transformations.

After three years (2008) a recurrence of the tumor in the

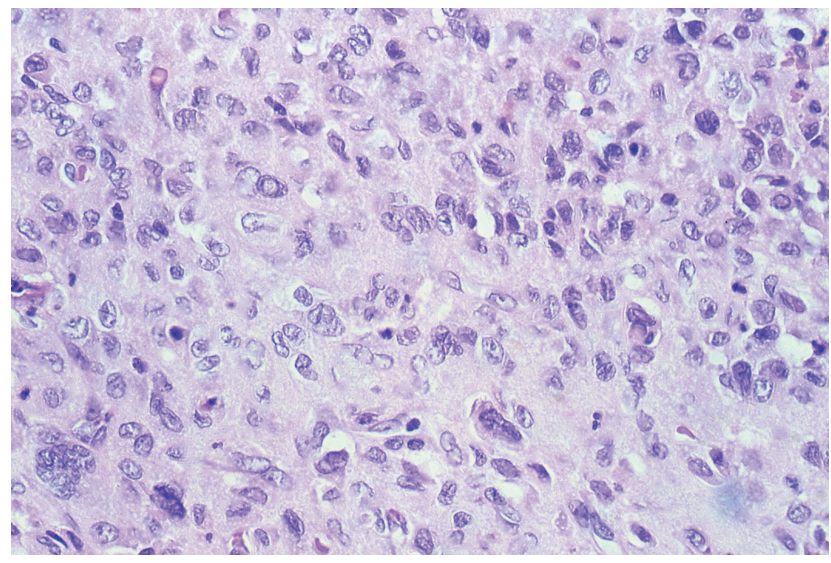

Fig. 1. Atypical menigeoma (Haematoxylin-Eosin [HE] stain). posterior horn of the left lateral ventricle was discovered on MRI Scans (Fig. 3A, B) at the same location as the tumor before (2002). Additionally, in this MRI another tumor located in the quadrigeminal lamina was detected (Fig. 4A, B). In 2009 , surgical resection of the tumor in the left lateral ventricle was performed. The pathological examination including immunohistochemical studies confirmed the diagnosis of a collision tumor consisting of components of a meningioma WHO II ${ }^{\circ}$ and a cavernoma (Fig. 5). The tumor cells stained positively for the epithelial membrane antigene (EMA) (Fig. 6) and the evidence of cavernoma components was shown by positive expression of CD-34 of the epithelial cells (Fig. 7). This exceptional diagnosis was confirmed by the German Brain Tumor Reference Center.

The postoperative MRI showed no residual tumor (Fig. 8A, B) and the tumor in the quadrigeminal lamina seemed to be regressing. Because of the benign characteristics of both tumor entities and the spontaneous regression of the tumor in the quadrigeminal lamina no adjuvant treatment was recommended. Up to the present, no evidence of tumor recurrence was seen in the MRI follow up.

\section{DISCUSSION}

As for meningiomas, they could arise anywhere as arachnoid cells are found and are usually slow growing, circumscribed, benign lesions. The WHO classification divides meningioma into three grades: grade I: benign meningioma,

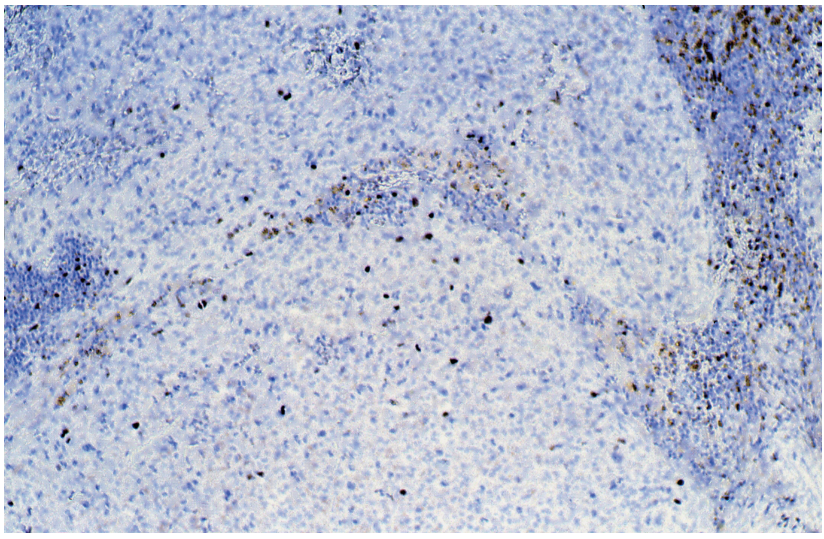

Fig. 2. Mib1-stain. 

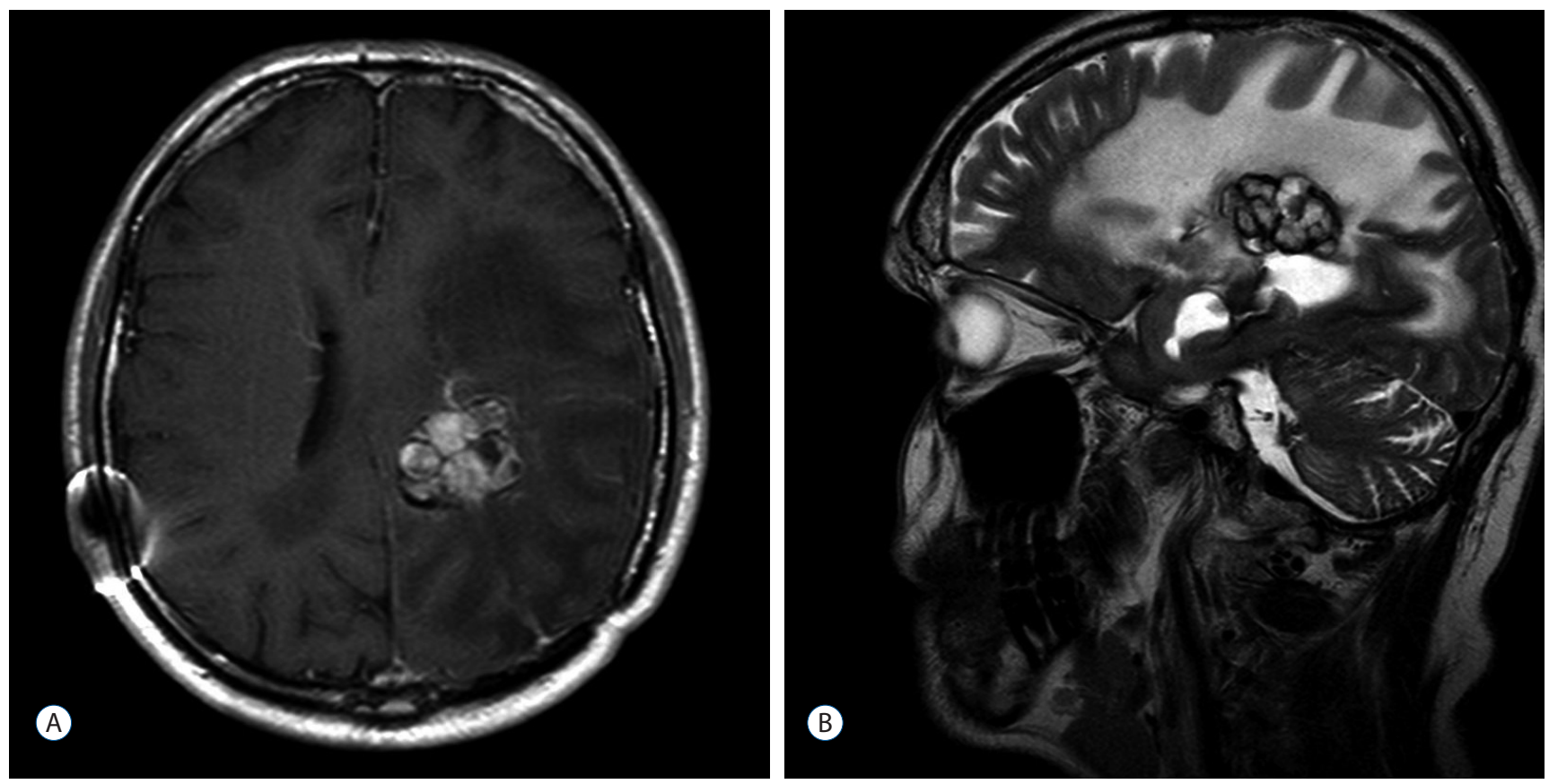

Fig. 3. A : T1-weighted axial MR image after contrast administration showing the tumor before the last operation. B : T2-weighted sagittal MR image showing the tumor before the last operation. MR : magnetic resonance.
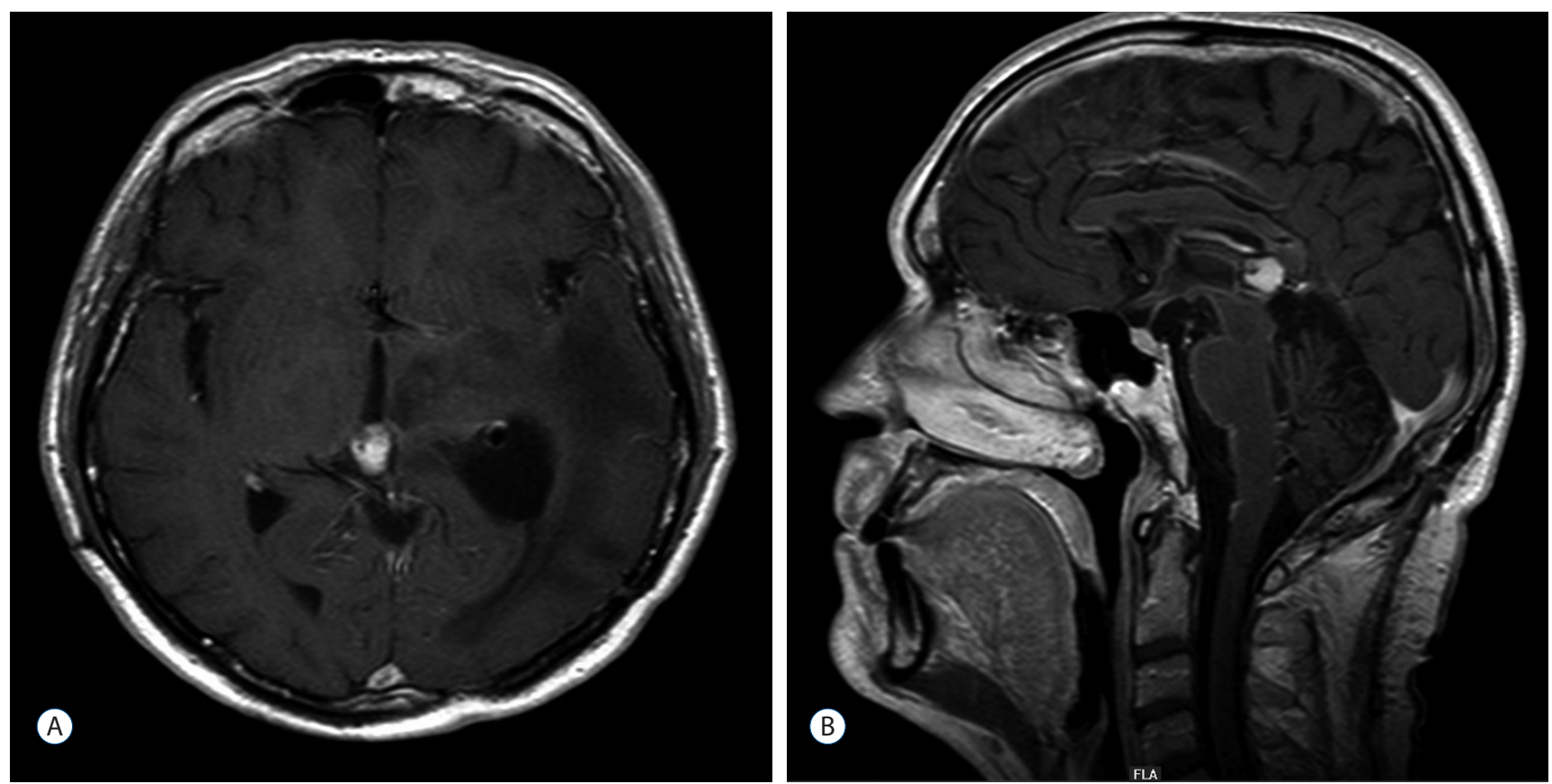

Fig. 4. A : T1 weighted axial MR image after contrast administration showing the tumor in the quadrigeminal lamina. B:T1 weighted sagittal MR image after contrast administration showing the tumor in the quadrigeminal lamina. MR : magnetic resonance.

grade II: atypical meningioma, grade III: malignant (anaplastic) meningioma ${ }^{5}$. They account for $14.3-19 \%$ of primary intracranial neoplasms; however, unlike nonmalignant tumors elsewhere in the body, these brain tumors can cause disability and may sometimes be life threatening ${ }^{4}$. The rates of recurrence after total tumor removal of benign $\left(\mathrm{WHO} \mathrm{I}^{\circ}\right)$,

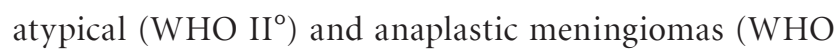
$\mathrm{III}^{\circ}$ ) were $6.9 \%, 34.6 \%$, and $72.7 \%$, respectively ${ }^{12)}$. When the 


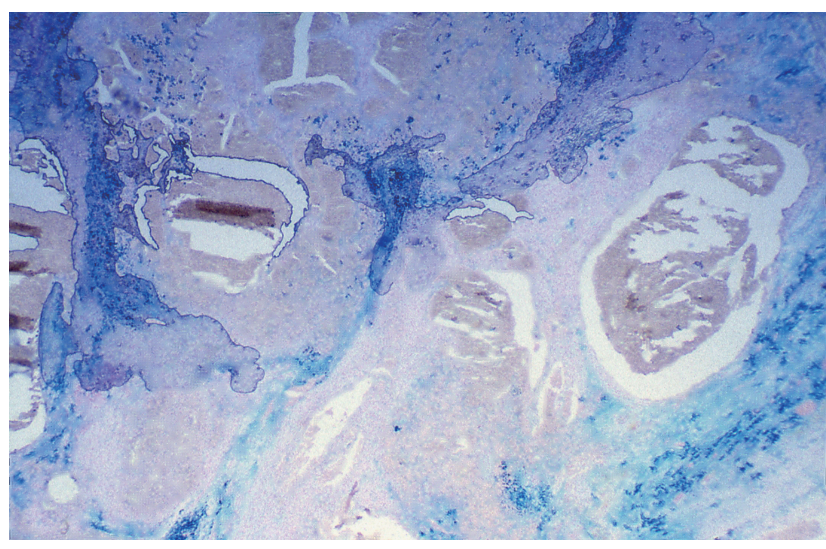

Fig. 5. Cavernoma. EVG stain. EVG : Elastica-van-Gieson.

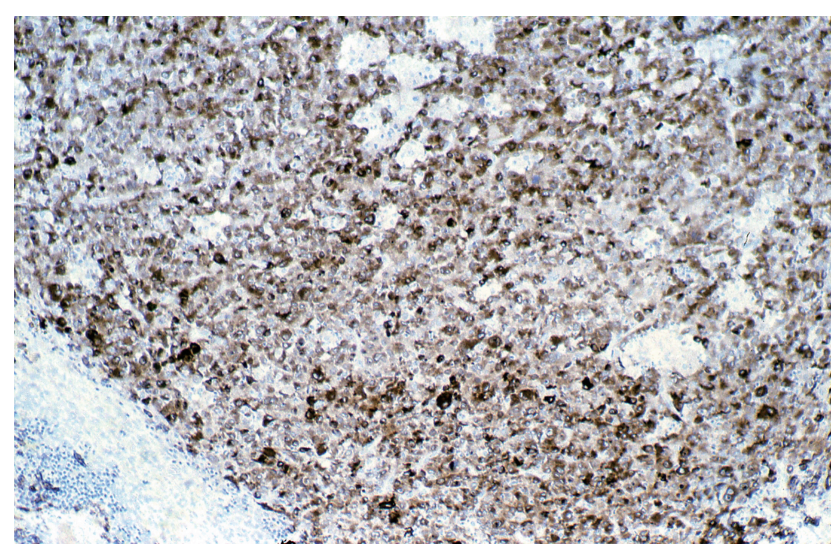

Fig. 6. EMA stain. EMA : epithelial membrane antigen.

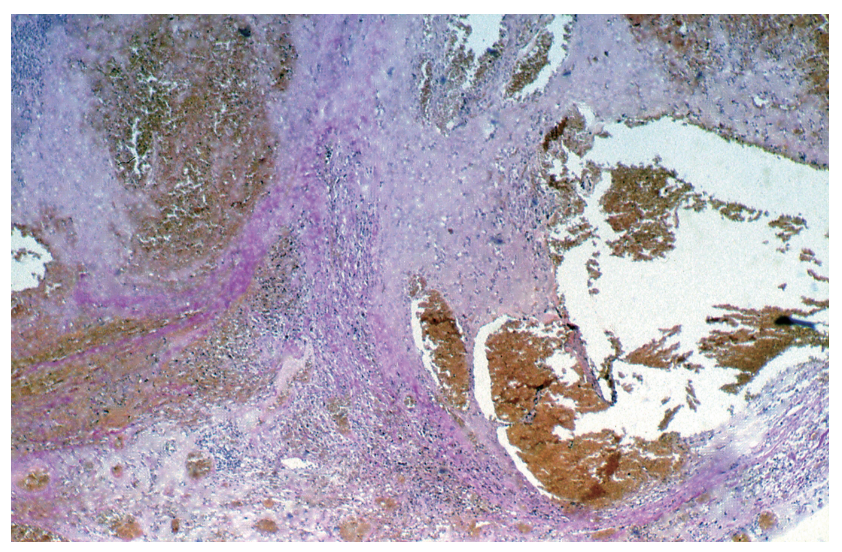

Fig. 7. Collision of Cavernoma and Meningeoma, showing positive staining for EMA and EVG stain. EMA : epithelial membrane antigen, EVG : Elasticavan-Gieson.

tumor removal is incomplete 5-year recurrence rates of $37-$ $85 \%$ are reported ${ }^{3}$. In our case, the first operation resulted in a complete removal of the meningioma. Seven years after the first procedure a recurrent tumor in the posterior horn of the left lateral ventricle was revealed.

In terms of cavernomas, they have several varying labels such as cavernous hemangioma, cavernous angioma, and cavernous malformation; the term cavernoma is often used when referring to presence in the brain. They are well-circumscribed, benign vascular hamartomas consisting of irregular thick and thin walled sinusoidal vascular channels located within the brain. They lack intervening neural parenchyma, large feeding arteries, or large draining veins; usually $1-5 \mathrm{~cm}$ in size and the prevalence of multiple occurrence varies between $3 \%$ and $50 \%$. They are filled with blood in various stages of thrombus formation. These lesions may arise de novo or after radiation ${ }^{4)}$. However, the patient in our case did not get any radiation therapy. Cavernous malformations can occur sporadically or follow an autosomal dominant pattern. Approximately half of them are familial in nature, and they occur multiply in 50\% of cases ${ }^{1)}$. In our case, the cavernoma appeared after the first removal of the meningioma in the fourth ventricle. After removal, there was no indication of another intracerebral cavernoma.

Without special or unique clinical features, collision tumors are difficult to diagnose preoperatively; pathological identification of the dual components is often the only way to ensure a correct diagnosis.

The pathological examination of the recurrent tumor in our case revealed the diagnosis of a collision tumor, consisting of meningioma and cavernoma components. Although this uncummon diagnosis was confirmed by the German Brain Tumor Reference Center, there is subject for debate. It seems to be difficult to make an unequivocal distinction between an angiomatous meningioma, which is an meningotheliomatous variety with closely packed blood vessels $\left(\mathrm{WHO} \mathrm{I}^{\circ}\right)^{4)}$, and the collision tumor, consisting of meningioma and cavernoma components. The pathological examination including immunohistochemical profile still supports this unique diagnosis.

In the literature, there are only approximately 50 reports of intracranial collision tumors. Most of them describe cases with collision tumors consisting of malignant astrocytoma (including glioblastoma) and meningioma ${ }^{3,6,7,9,10)}$. There are 

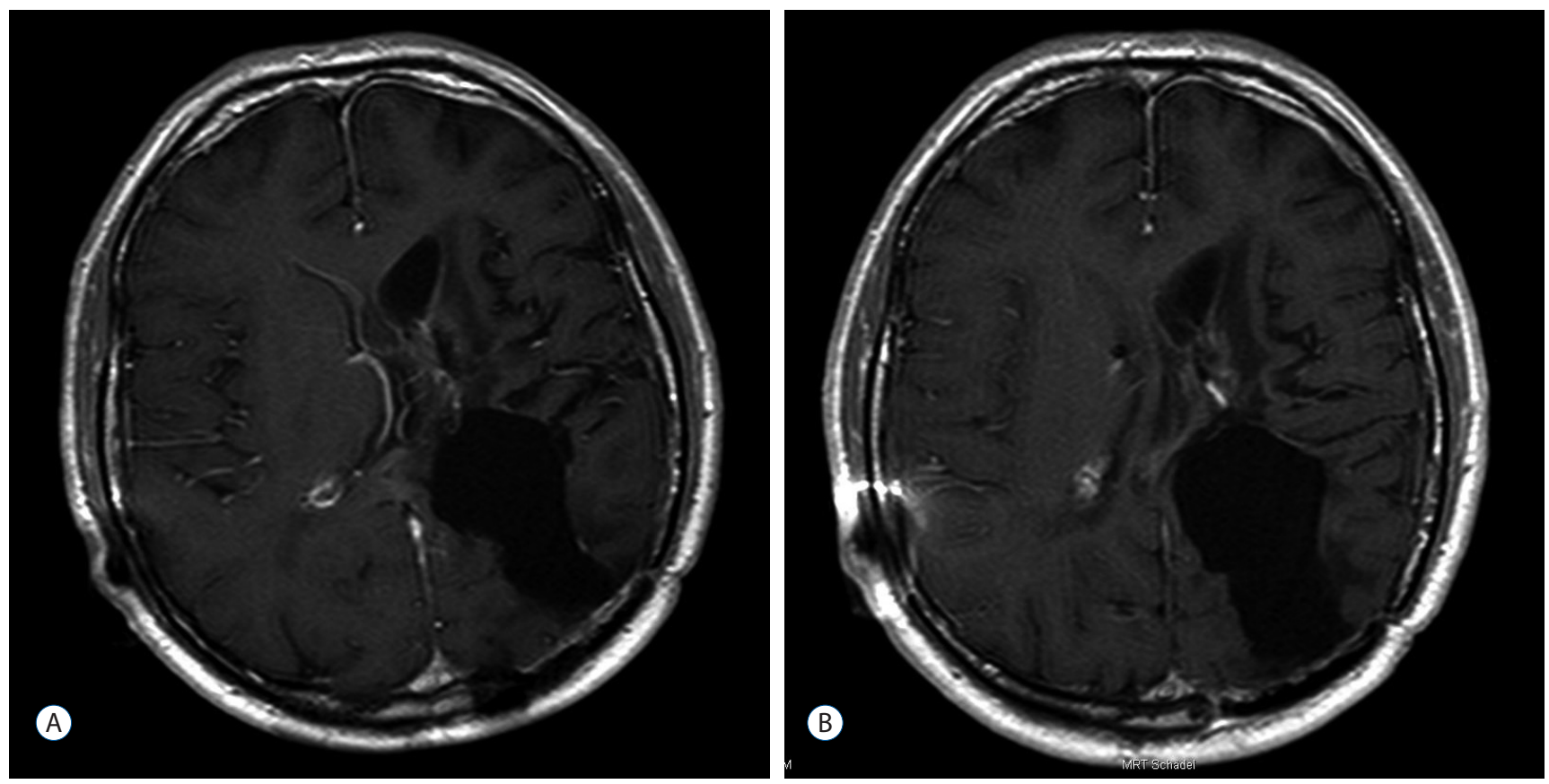

Fig. 8. A : T1 weighted axial MR image after contrast administration after tumor extirpation. B : T1 weighted axial MR image after contrast administration, three year follow up. MR: magnetic resonance.

also reports of collision tumors consisting of two different cerebral metastases ${ }^{2,8)}$. In almost all of these cases, the collision tumor consists of at least one malignant entity, but in our case, the collision tumor consists of two benign entities. However, we must consider that one entity is an atypical meningioma $\left(\mathrm{WHO} \mathrm{II}^{\circ}\right.$ ). Interestingly, the meningioma, which potentially can evolve malignancy and malignant growth patterns, was a recurrent tumor after total removal seven years before. Thus, invasive proliferation does not appear to be the reason for the collision.

Our unusual case of a collision tumor consisting of meningioma and cavernoma seems to be a unique collision tumor of the brain. To our knowledge, this is the first reported case of a collision tumor of the brain consisting of two benign entities (meningioma and cavernoma components). Both tumors were located in the ventricular system. We hypothesize, that a possible explanation for the collision of the two different tumors may be a cerebrospinal fluid mediated migration of tumor cells. Until now, there is no sign of any tumor recurrence after 5-years of follow-up; therefore, the surgical tumor removal without adjuvant therapy seems to be the treatment of choice.

\section{CONCLUSION}

We hypothesize, that a possible explanation for the collision of the two different tumors in the ventricular system may be tumorcell migration mediated by the cerebrospinal fluid.

Until now, there is no sign of any tumor recurrence in a 5 -years follow up. Therefore surgical tumor removal without adjuvant therapy seems to be the treatment of choice.

\section{References}

1. Cavalcanti DD, Kalani MY, Martirosyan NL, Eales J, Spetzler RF, Preul MC : Cerebral cavernous malformations: from genes to proteins to disease. J Neurosurg $116: 122-132,2012$

2. Dewan $S$, Alvarez VE, Donahue JE, Cielo D : Intracranial collision metastases of prostate and esophageal carcinoma. J Neurooncol 95 : 147150, 2009

3. Drlicek M1, Aichholzer M, Wurm G, Bodenteich A, Fischer J : Collisiontumour composed of glioblastoma and meningioma-a case report. Pathologe 25 : 402-405, 2004

4. Greenberg MS : Handbook of neurosurgery, ed 6. Tampa: Greenberg Graphics, Inc., 2006, pp426-429, pp841-843 
Collision Tumor Composed of Meningioma and Cavernoma | Weigel J, et al.

5. Maier $H$, Ofner D, Hittmair A, Kitz K, Budka $H$ : Classic, atypical, and anaplastic meningioma: three histopathological subtypes of clinical relevance. J Neurosurg 77 : 616-623, 1992

6. Mitsos AP, Konstantinou EA, Fotis TG, Lafazanos SA, Kontogeorgos G, Georgakoulias NV : Sphenoid wing meningioma and glioblastoma multiforme in collision - case report and review of the literature. Neurol Neurochir Pol 43 : 479-483, 2009

7. Nestler U, Schmidinger A, Schulz C, Huegens-Penzel M, Gamerdinger UA, Koehler $A$, et al. : Glioblastoma simultaneously present with meningioma-report of three cases. Zentralbl Neurochir 68 : 145-150, 2007

8. Palka KT, Lebow RL, Weaver KD, Kressin MK : Intracranial collision metastases of small-cell lung cancer and malignant melanoma. J Clin
Oncol 26 : 2042-2046, 2008

9. Prayson RA, Chowdhary S, Woodhouse S, Hanson M, Nair S : Collision of a syncytial meningioma and malignant astrocytoma. Ann Diagn Pathol 6 : 44-48, 2002

10. Vaquero J, Coca $S$, Martínez $R$, Jiménez $C$ : Convexity meningioma and glioblastoma in collision. Surg Neurol 33 : 139-141, 1990

11. Willis RA : Structure and growth of tumors. Pathology of tumors, ed 4. London : Butterworth, 1967, pp138

12. Wrobel G, Roerig P, Kokocinski F, Neben K, Hahn M, Reifenberger G, et al. : Microarray-based gene expression profiling of benign, atypical and anaplastic meningiomas identifies novel genes associated with meningioma progression. Int J Cancer 114 : 249-256, 2005 